\title{
A Multi-Flock Approach to Rapid Dynamic Generator Coherency Identification
}

\author{
Jin Wei and Deepa Kundur \\ The Edward S. Rogers Sr. Department of Electrical and Computer Engineering \\ University of Toronto \\ \{jinwei, dkundur\}@.comm.utoronto.ca
}

\begin{abstract}
In cases of intentional fault and cyber attack of smart grid systems, the reaction-time to mitigate disaster is small. Moreover, the large-scale impact of such disruptions is often in the form of inter-area oscillations. To address these issues, we propose a novel multi-flock-based technique to identify generator coherence within a short observation window suitable for mitigation approaches in smart grid systems. We present our approach and demonstrate its effectiveness in identifying generator clustering quickly for different fault scenarios of the 39-bus New England test system.
\end{abstract}

Index Terms-Generator coherence identification, flocking models.

\section{INTRODUCTION}

As we shift towards an era of "smarter" power systems, we witness the increased functionality and improved consumercentricity of energy delivery networks. These cyber-physical systems can be distinguished from their legacy counterparts, in part, through their ability to participate in high granularity short-time scale informational and financial transactions. Such diminished time scales are also characteristic of the anticipated physical interactions within smart power systems. Moreover, the physical components, increasingly operated under stress in this environment of market deregulation, can exhibit possible inter-area oscillations reducing power quality.

In this paper, we develop a coherency identification algorithm for smart power systems that works rapidly and with limited data sets to detect generator clustering in the presence of inter-area oscillations from system faults and cyberphysical attack. Knowledge of emerging generator groups is an essential assumption upon which most effective corrective actions are based. Moreover, the decreasing time scales of operation necessitate that generator group identification be conducted with low latency. Building upon our past success with flocking-based models [1]-[3], our approach, designed with real-time considerations, makes use of a flocking strategy for coherence identification.

The next section provides a brief background on generator coherence and its identification. We introduce the concepts of flocking and multiple flocks. In Section II, we discuss our proposed scheme. Simulation results are presented in Section III followed by final remarks in Section IV.

\section{A. Generator Coherency Identification}

Inter-area oscillations are a complex phenomenon observed between interconnected synchronous generators in power sys- tems. These oscillations, associated with groups of generators that swing against each other at frequencies below $1 \mathrm{~Hz}$, are intrinsically nonlinear and dynamic [4]. Most strategies to dampen or counteract such modes of operations require that the groups of coherent generators be first identified. The efficient and accurate generator coherency determination is a critical element of maintaining transient stability especially in the context of wide are monitoring. Numerous existing techniques have been developed for modern power systems.

For example, Xu and Vittal [5] propose a topological graphbased approach to identify generator coherence. The approach, implemented offline, exhibits high accuracy and is suitable for the study of planned islanding strategies that maintain system stability. In contrast, the authors of [6]-[8] develop on-line methods to identify generator coherency by applying signal processing techniques such as Fourier, Hilbert-Huang and Arnoldi transform analyses. The approaches do not employ explicit models of the system requiring that sufficient data samples with specific sampling data patterns be available.

We argue that the emerging smart grid will require rapid response to inter-area oscillations requiring real-time generator coherency identification using limited sample data. In order to meet these objectives, we make use of a model-based approach. In contrast to techniques that account for grid topology, which often lead to complex solutions, we employ flocking theory as a metaphor for coherent generator clustering. The synchronous generators are each modeled as birdlike objects (termed boids [9]). The relative phase, frequency and acceleration of the generators influence inter-boid forces associated with flocking theory such that those boids deemed to be part of the same flock correspond to generators in the same coherent cluster and those part of different flocks relate to generators of distinct clusters. The inter-boid movements over time enable the visual identification of coherent clusters.

\section{B. Flocking}

Flocking is a behavior exhibited by groups of birds participating in a shared objective such as flying to a mutual destination. Such behavior enables a collective to accomplish an objective difficult to achieve individually through a combination of cooperation, consensus and informed-adaptation.

The behavior within a single flock has been described by a set of heuristic agent-interaction rules that we employ in this 
work [9], [10]:

1) flock centering: boids attempt to stay close to nearby flockmates;

2) velocity matching: boids attempt to match velocity with nearby flockmates;

3) collision avoidance: boids avoid collisions with nearby flockmates; and

4) obstacle avoidance: boids avoid obstacles often steering away from their goals.

We have recently applied these principles to the problem of modeling the dynamics required for transient stabilization of faulted smart grid systems [1]. Here, an effective analogy between the dynamics of generator synchronization and that of flocking is studied. A distributed cyber-physical control approach is developed such that in the face of faults and attacks, the system is re-stabilized by "steering" its dynamics as a cohesive flock. To reduce the communications and energy overhead of such an approach, the natural coherence of the generators is leveraged.

Given the success of this flocking-based metaphor of coupled synchronous generators, in this paper we aim to develop a technique to detect generator coherence by modeling the generator characteristics as the interactions of boids in multiple flocks. Those generators in the same coherent group would correspond to boids of the same flock. Thus, we extend our flocking paradigm to include the characterization of multiple species flocking via a feature similarity rule. Here, a flock boid tries to stay close to those boids with "similar features" and stays apart from boids with dissimilar features much like the flock separation observed for different species in nature.

\section{Multi-Flock Coherency Identification}

We assume that each generator has a phasor measurement unit (PMU) that acquires its phase and frequency information and communicates it to a control center for modeling and decision-making.

\section{A. Multi-Flock Modeling}

We assume there are $C$ generators (boids) indexed as $i=$ $1,2, \ldots, C$. Each boid carries three-dimensional information describing the generator's status at time $t=k$ as follows:

$$
\left\{\begin{array}{l}
\mathcal{I}_{i}^{1}(k)=\theta_{i}(k) \\
\mathcal{I}_{i}^{2}(k)=\omega_{i}(k) \\
\mathcal{I}_{i}^{3}(k)=\delta_{i}(k)
\end{array},\right.
$$

where $\mathcal{I}_{i}(k)=\left[\mathcal{I}_{i}^{1}(k) \mathcal{I}_{i}^{2}(k) \mathcal{I}_{i}^{3}(k)\right]^{T}, \theta_{i}(k)$ and $\omega_{i}(k)$ are the phase angle and the normalized frequency, respectively, of the $i$ th generator at the time step $t=k$ that are obtained directly from PMU information, and $\delta_{i}(k)$ is the acceleration of the $i$ th generator at the time step $t=k$ estimated from the current and historical values of $\omega_{i}(k)$.

The state of each boid is described as follows:

$$
\mathcal{S}_{i}(k)=\left[\mathbf{p}_{i}(k), \mathbf{v}_{i}(k)\right]^{T},
$$

where $\mathbf{p}_{i}(k), \mathbf{v}_{i}(k) \in \mathbb{R}^{2 \times 1}$ denote the boid's position and the velocity, respectively. Two boids are considered to be neighbors at time step $t=k$ if the distance between them is less than the predetermined threshold $d_{c}$. Therefore, we define the set of neighbors for the $i$ th boid as follows: $\mathcal{N}_{i}(k)=\left\{\forall j \mid\left\|\mathbf{p}_{i}(k)-\mathbf{p}_{j}(k)\right\|<d_{c}\right\}$.

1) Feature Similarity: We compute the informational (feature) similarity between neighboring Boids $i$ and $j$ as follows. For $j \in \mathcal{N}_{i}(k)$,

$$
\zeta_{i j}(k)=\left|\sum_{n=1}^{3} \alpha_{n} \times\left(\mathcal{I}_{i}^{n}(k)-\mathcal{I}_{j}^{n}(k)\right)\right|,
$$

where $\left\{\alpha_{n}\right\}$ is a scalar weight determining the impact of specific information on boid interaction. This metric is compared to the following dynamic threshold:

$$
\zeta_{t h}(k)=\frac{\beta}{C} \sum_{i=1}^{C} \sum_{j \in \mathcal{N}_{i}(k)} \zeta_{i j}(k),
$$

where $\beta$ is a parameter to determine whether Boids $i$ and $j$ are part of the same flock at $t=k$. If $\zeta_{i j}(k) \leq \zeta_{t h}(k)$ they are assumed to be in the same flock and hence are called flockmates. If $\zeta_{i j}(k)>\zeta_{t h}(k)$, they are assumed to be in different flocks.

2) Intra-Flock Centering and Collision Avoidance: Flock centering and collision avoidance involves the attraction of boids to one another such that they are in the same geographic proximity but are non-overlapping. We model this for boids within the same flock through inter-boid forces that depend on relative distance and informational similarity. Thus, for $\zeta_{i j}(k) \leq \zeta_{t h}(k)$, Boids $i$ and $j$ are assumed to exhibit cohesion; they are attracted to one another up to a desired position distance $d^{*}$ (at which point we consider the attraction strength to be zero) and then if any closer slightly repel each other to avoid collision. The higher informational similarity between the boids, the stronger (weaker) the strength (repulsion) when their distance is above (below) $d^{*}$; as $\zeta_{i j}(k) \rightarrow 0$ this interaction approaches zero.

We design the following potential energy function:

$$
\begin{aligned}
V_{1}(k)= & \sum_{i=1}^{C} \sum_{j \in \mathcal{N}_{i}(k)}\left\{\frac { \gamma _ { 1 } } { \gamma _ { 2 } } \operatorname { e x p } \left[\gamma _ { 2 } ( \zeta _ { i j } ( k ) - \zeta _ { t h } ( k ) ) \left(\| \mathbf{p}_{i}(k)\right.\right.\right. \\
& \left.\left.-\mathbf{p}_{j}(k)\left\|_{\sigma}-\right\| d^{*} \|_{\sigma}\right)\right]-\gamma_{1} \times\left(\zeta_{i j}(k)-\zeta_{t h}(k)\right) \\
& \left.\left(\left\|\mathbf{p}_{i}(k)-\mathbf{p}_{j}(k)\right\|_{\sigma}-\left\|d^{*}\right\|_{\sigma}\right)\right\}
\end{aligned}
$$

where $\|\cdot\|_{\sigma}$ denotes the $\sigma$-norm $\|\mathbf{x}\|_{\sigma}=\frac{1}{\epsilon}(\sqrt{1+\epsilon\|\mathbf{x}\|}-1)$, and $\epsilon, \gamma_{1}$ and $\gamma_{2}$ are positive parameters.

Using Eq. (5), we calculate the acceleration caused by the potential energy function $V_{1}(k)$ to give:

$$
\begin{aligned}
\mathbf{g}_{i, 1}(k)= & Y \gamma_{1} \times\left(\zeta_{i j}(k)-\zeta_{t h}(k)\right)\left\{\operatorname { e x p } \left[\gamma_{2}\left(\zeta_{i j}(k)-\zeta_{t h}(k)\right)\right.\right. \\
& \left.\left.\left(\left\|\mathbf{p}_{i}(k)-\mathbf{p}_{j}(k)\right\|_{\sigma}-\left\|d^{*}\right\|_{\sigma}\right)\right]-1\right\} \mathbf{n}_{i j}
\end{aligned}
$$


where

$$
\mathbf{n}_{i j}=\frac{\mathbf{p}_{j}(k)-\mathbf{p}_{i}(k)}{\sqrt{1+\epsilon\left\|\mathbf{p}_{j}(k)-\mathbf{p}_{i}(k)\right\|^{2}}} .
$$

3) Velocity Matching: In a flock, flockmates typically demonstrate alignment of their velocities. It is well established that this can be modeled as a distributed consensus problem in which the boids represent the vertices of a graph and the associated edge weights determine the degree of interaction to achieve alignment [11]. We design the adjacency matrix elements of such a graph in our formulation as:

$$
a_{i j}(k)=\rho_{h}\left(\frac{\left\|\mathbf{p}_{i}(k)-\mathbf{p}_{j}(k)\right\|_{\sigma}}{\left\|d_{c}\right\|_{\sigma}}\right),
$$

for $j \in \mathcal{N}_{i}(k)$ where

$$
\rho_{h}(x)= \begin{cases}1, & z \in[0, h) ; \\ \frac{1}{2}[1+\cos (\pi(x-h) /(1-h))], & z \in[h, 1] ; \\ 0, & \text { otherwise. }\end{cases}
$$

The corresponding acceleration introduced is:

$$
\mathbf{g}_{i, 2}(k)=\sum_{j \in \mathcal{N}_{i}(k)} a_{i j}(k)\left(\mathbf{p}_{j}(k)-\mathbf{p}_{i}(k)\right) .
$$

4) Obstacle Avoidance: For $\zeta_{i j}(k)>\zeta_{t h}(k)$, Boids $i$ and $j$ are considered to be dissimilar and therefore in different flocks. Their interaction is modeled as a mutual avoidance in which each considers the other to be an obstacle in their flock's trajectory. We model the corresponding repulsion strength to be dependent on their distance relative to one another; the higher the dissimilarity the stronger the repulsion up to a distance threshold $d_{r}$ at which point their repulsion strength decreases to 0 .

We design the associated acceleration as:

$$
\begin{aligned}
\mathbf{g}_{i, 3}(k) & =\rho_{h} \mathbf{n}_{i k}\left(\left\|\mathbf{p}_{i}(k)-\mathbf{p}_{j}(k)\right\|_{\sigma} /\left\|d_{r}\right\|_{\sigma}\right)\left(\zeta_{t h}(k)-\zeta_{i j}(k)\right) \\
& \left(\left\|\mathbf{p}_{i}(k)-\mathbf{p}_{j}(k)\right\|_{\sigma}-\left\|d_{r}\right\|_{\sigma}\right)\left[\epsilon \times\left(\zeta_{t h}(k)-\zeta_{i j}(k)\right)^{2}\right. \\
& \left.\left(\left\|\mathbf{p}_{i}(k)-\mathbf{p}_{j}(k)\right\|_{\sigma}-\left\|d_{r}\right\|_{\sigma}\right)^{2}+1\right]^{-\frac{1}{2}} .
\end{aligned}
$$

\section{B. Coherent Generator Identification}

Thus the dynamics of Boid $i$ can be modeled as follows:

$$
\left\{\begin{array}{l}
\mathbf{v}_{i}(k+1)=\mathbf{v}_{i}(k)+\Delta t \sum_{l=1}^{3} w_{l} \mathbf{g}_{i, l}(k), \\
\mathbf{p}_{i}(k+1)=\mathbf{p}_{i}(k)+\Delta t \mathbf{v}_{i}(k),
\end{array}\right.
$$

where $\Delta t$ is the algorithm time step for coherence identification, $w_{l}$ denotes the weight representing the impact of the component $\mathbf{g}_{i, l}$ on the acceleration, and the accelerations $\mathbf{g}_{i, l}$ are given by Eqs. (6), (10) and (11) for $l=1,2,3$, respectively.

The state of each boid $\mathcal{S}_{i}(k)=\left[\mathbf{p}_{i}(k), \mathbf{v}_{i}(k)\right]^{T}$ is used to plot its trajectory in time during the observation period. From this graph, we can easily obtain the clustering results of the boids indicating coherent clusters of generators. We demonstrate in the next section how our models effectively determine coherent generator clusters under a variety of faults.

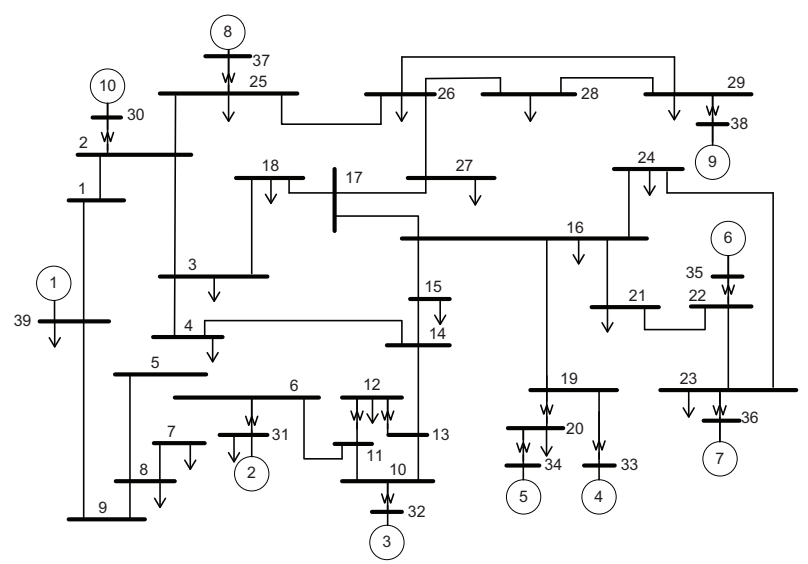

Fig. 1. New England 39-bus power system,

\section{Simulation Results}

Our test system consists of the 39-bus New England test system of Fig. 1 consisting of $C=10$ generators, which we simulate with MATLAB/Simulink. We evaluate the efficiency of our proposed multi-flock-based dynamic generator coherency determination scheme for three cases representing different fault locations and for PMU sampling rates of $50 \mathrm{~Hz}$ and $100 \mathrm{~Hz}$. The dynamics of the boids is given by Eq. (12) with initial locations randomly generated within a unit area square. The parameters are set as follows: $d_{c}=10, d^{*}=0.05$, $d_{r}=8, \beta=2, \gamma_{1}=1, \gamma_{2}=0.5, \epsilon=1, h=1$ and $\delta t=0.02$ for a PMU rate of $50 \mathrm{~Hz}$ and $\delta t=0.01$ for a PMU rate of $100 \mathrm{~Hz}$. In addition we set the weights in Eq. (3) to $\alpha_{1}=0.01$, $\alpha_{2}=80$, and $\alpha_{3}=5000$, and the weights of Eq. (12) as $w_{1}=5000, w_{2}=1500$, and $w_{3}=2000$.

\section{A. Case I}

We assume that a 3-phase fault occurs at Bus 14 at $t=0 \mathrm{~s}$ and the associated Line $14-15$ is opened at time $t=0.3 \mathrm{~s}$, postcritical clearing time (CCT). The generators' normalized rotor frequencies and phase angles over a period of $5 \mathrm{~s}$ are shown in Fig. 2. We observe that the generator coherency involving the following groups: $\left\{G_{1}\right\},\left\{G_{2}, G_{3}\right\}$, and $\left\{G_{4}, G_{5}, \ldots, G_{10}\right\}$. The corresponding void trajectories introduced by our flocking analogy are presented in Fig. 3 for a very brief observation period of $t=0.35 \mathrm{~s}$ assuming PMU sampling rates of $100 \mathrm{~Hz}$ and $50 \mathrm{~Hz}$. The arrows on each trajectory indicates the velocity direction of each boid. From Fig. 3, we can identify the boids associated with $G_{2}$ and $G_{3}$ traveling together as well as those associated with $G_{4}, G_{5}, \ldots, G_{10}$. Thus, we observe that the separate flocks corresponding to the observed coherent generator groups.

\section{B. Case II}

We consider a 3-phase fault to occur at Bus 21 at $t=0$. The associated Line 21-22 is opened at $t=0.3 \mathrm{~s}$, again after the CCT. The generators' normalized rotor frequencies and phase angles over a period of $5 \mathrm{~s}$ are shown in Fig. 4 giving the following coherent clusters groups: $\left\{G_{1}\right\},\left\{G_{6}, G_{7}\right\}$, and $\left\{G_{2}, G_{3}, G_{4}, G_{5}, G_{8}, G_{9}, G_{1} 0\right\}$. The corresponding boid trajectories for a very brief observation period of $t=0.35 \mathrm{~s}$ 

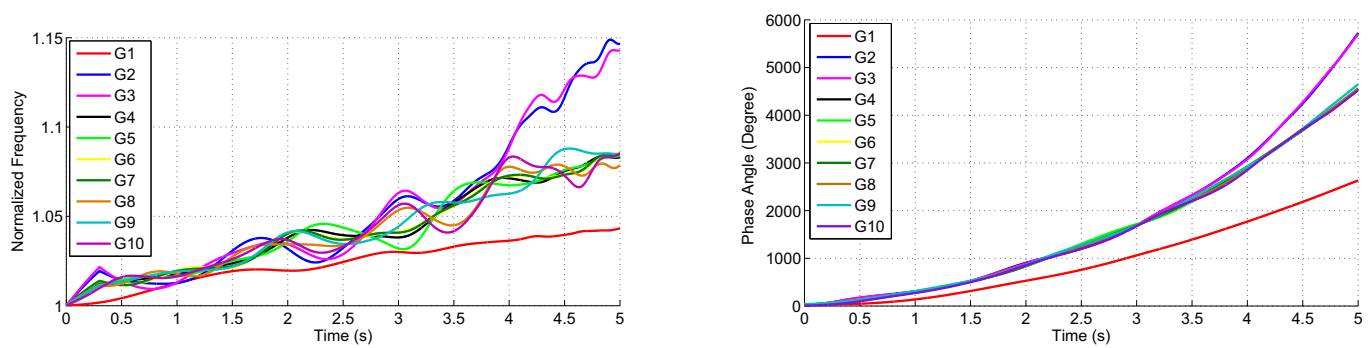

Fig. 2. Normalized rotor frequencies and phase angles versus time.
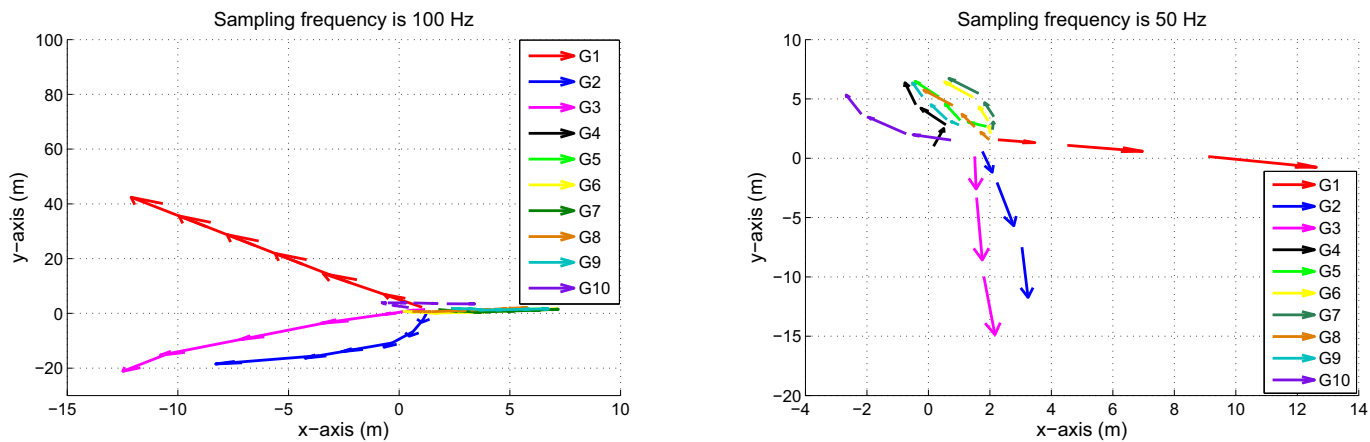

Fig. 3. The trajectories of the boids by using the PMUs with $100 \mathrm{~Hz}$ and $50 \mathrm{~Hz}$.

and PMU sampling rates of $100 \mathrm{~Hz}$ and $50 \mathrm{~Hz}$ are shown in Fig. 5. As evident, the void associated with $G_{1}$ travels away from the others that form two groups, one corresponding to $G_{6}$ and $G_{7}$, and the other to $G_{2}, G_{3}, G_{4}, G_{5}, G_{8}, G_{9}$ and $G_{1} 0$.

\section{Case III}

Next, we consider a 3-phase fault to occur at Bus 2 at $t=0 \mathrm{~s}$ and the associated Line $2-25$ is opened at time $t=0.3 \mathrm{~s}$ (again, after the CCT). The generators' normalized rotor frequencies and phase angles over a period of $10 \mathrm{~s}$ are shown in Fig. 6 which show the following coherent groupings: $\left\{G_{1}\right\},\left\{G_{10}\right\}$, $\left\{G_{8}, G_{9}\right\}$, and $\left\{G_{2}, G_{3}, G_{4}, G_{5}, G_{6}, G_{7}\right\}$. Fig. 7 shows the flock trajectories at time $t=0.35 \mathrm{~s}$ for PMU sampling rates of $100 \mathrm{~Hz}$ and $50 \mathrm{~Hz}$, once again consistent with the generator clusters.

\section{FinAl REMARKS}

We present a novel multi-flocking based approach to realtime generator coherency identification that requires a much shorter observation window than existing approaches. We present three case studies to demonstrate the accuracy of the approach in identifying generator clustering. Our results imply that the higher the PMU sampling rate the greater the flock separation although both sampling rates of $100 \mathrm{~Hz}$ and $50 \mathrm{~Hz}$ provide a good visual separation for determining clusters rapidly.

\section{ACKNOWLEDGEMENTS}

Research funding was provided by the U.S. National Science Foundation under grant ECCS-1028246 and the Nor- man Hackerman Advanced Research Program Project Number 000512-0111-2009.

\section{REFERENCES}

[1] J. Wei, D. Kundur, T. Zourntos, and K. Butler-Purry, "A flocking-based dynamical systems paradigm for smart power system analysis," in Proc. IEEE Power \& Energy Society General Meeting, San Diego, California, July 2012.

[2] J. Wei and D. Kundur, "Two-tier hierarchical cyber-physical security analysis framework for smart grid," in Proc. IEEE Power \& Energy Society General Meeting, San Diego, California, July 2012.

[3] _ - "A flocking-based model for dos-resilient communication routing in smart grid," in Proc. IEEE Global Communications Conference (GLOBECOM), Anaheim, CA, December 2012.

[4] M. Klein, G. Rogers, and P. Kundur, "A fundamental study of inter-area oscillations in power systems," IEEE Transactions on Power Systems, vol. 6, no. 3, pp. 914-921, August 1991.

[5] G. Xu and V. Vittal, "Slow coherency based cutset determination algorithm for large power systems," IEEE Transactions on Power Systems, vol. 26, no. 2, pp. 877-884, May 2010.

[6] M. Jonsson, M. Begovic, and J. Daalder, "A new method suitable for real-time generator coherency determination," IEEE Transactions on Power Systems, vol. 19, no. 3, pp. 1473-1482, August 2004.

[7] N. Senroy, "Generator coherency using the hilbert-huang transform," IEEE Transactions on Power Systems, vol. 23, no. 4, pp. 1701-1708, November 2008.

[8] Y. Susuki and I. Mezić, "Nonlinear koopman modes and coherency identification of coupled swing dynamics," IEEE Transactions on Power Systems, vol. 26, no. 9, pp. 1894-1904, 2011.

[9] C. Reynolds, "Flocks, herds, and schools: a distributed behavioral model," Computer Graphics, vol. 21, no. 4, pp. 25-34, July 1987.

[10] I. Couzin, J. Krause, R. James, G. Ruxton, and N. Franks, "Collective memory and spatial sorting in animal groups," in Journal of Theoretical Biology, 2002

[11] R. Olfati-Saber, "Flocking for multi-agent dynamic systems: Algorithms and theory," IEEE Transactions on Automatic Control, vol. 51, no. 3 , pp. 401-420, March 2006. 

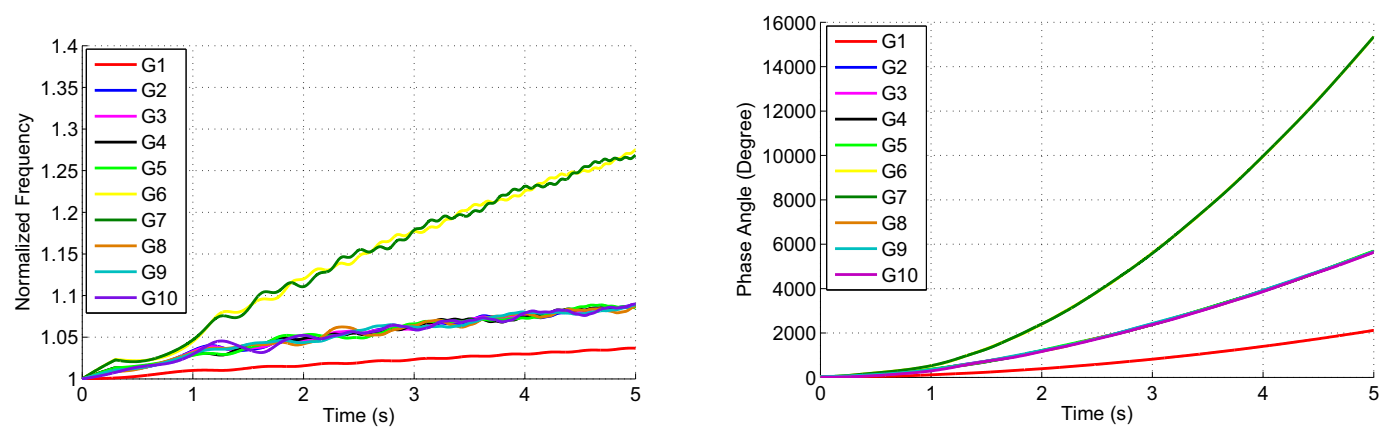

Fig. 4. Normalized rotor frequencies and phase angles versus time.
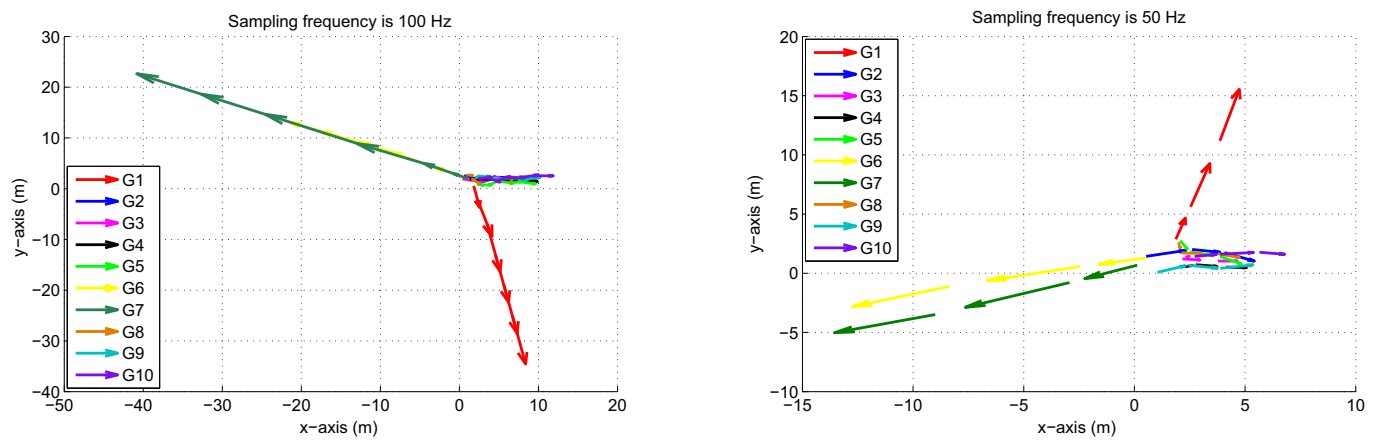

Fig. 5. The trajectories of the boids by using the PMUs with $100 \mathrm{~Hz}$ and $50 \mathrm{~Hz}$.
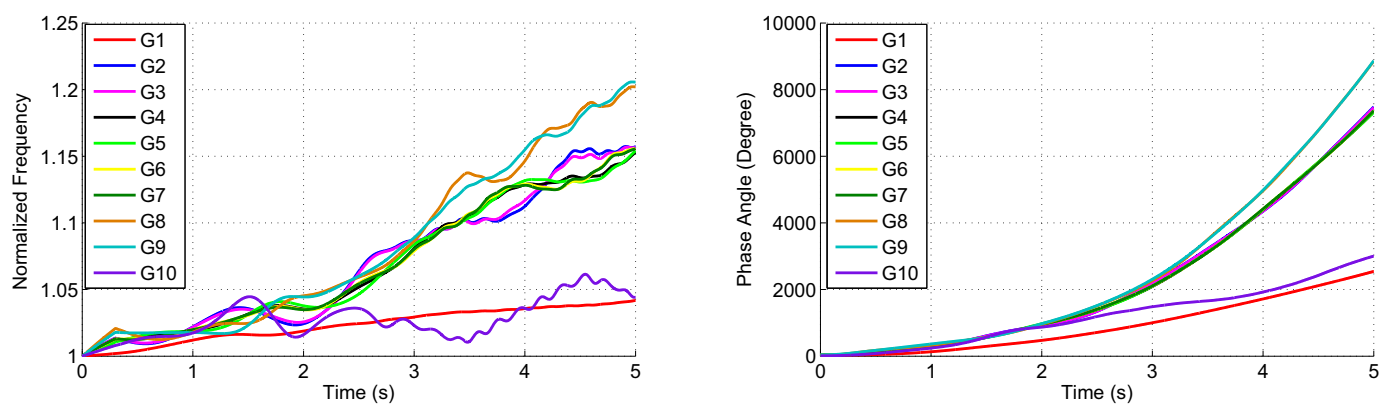

Fig. 6. Normalized rotor frequencies and phase angles versus time.
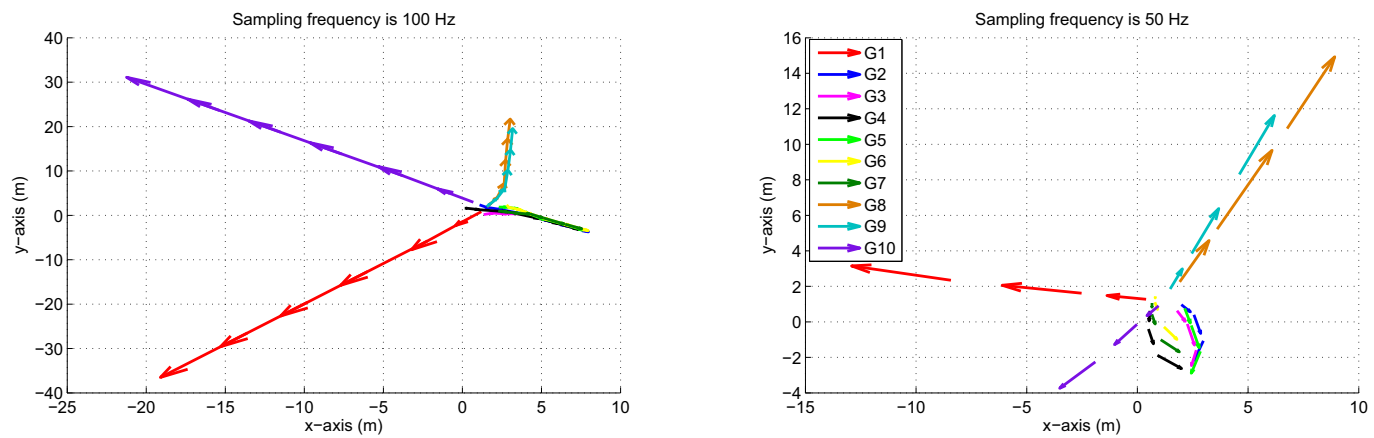

Fig. 7. The trajectories of the boids by using the PMUs with $100 \mathrm{~Hz}$ and $50 \mathrm{~Hz}$. 